EGG-EP-10715

(DE93011851)

Distribution Category UC-249

\title{
AIR CLASSIFICATION THEORY AND DESIGN FOR MUNICIPAL SOLID WASTE PROCESSING
}

Final Report

April 1993

Work Performed Contract DE-AC07-761D01570

\author{
Prepared for the \\ U.S. Department of Energy \\ Under DOE Idaho Field office \\ Sponsored by the Office of the Assistant Secretary \\ for Conservation and Renewable Energy \\ Office of Industrial Technologies \\ Washington, D.C. \\ Prepared by \\ Department of Civil and Environmental Engineering \\ Duke University \\ Durham, North Carolina 27706
}


Table of contents

Page

List of Exhibits i

I. An Introduction to Air Classification for

Municipal Solid Waste Processing and

Objectives of This Research . . . . . . . . . . . . . 1

II. Development of Theory and Design:

Historical Perspective . . . . . . . . . . . . . 2

III. Laboratory Equipment, Procedures and

Test Protocol . . . . . . . . . . . . . . . . . 10

IV. Results and Discussion . . . . . . . . . . . . . 20

v. Conclusions of the Research . . . . . . . . . . . 33

Appendix I: Bibliography

Appendix II: Notation 


\section{List of Exhibits}

Exhibit

Title

Page

1 Air Classifier Throat Configurations 3

2 Spheres Falling from Rest 8

3 Air classifier system1 12

4 Air Velocity Graph: Symmetric Wave Form 15

5 Passive Air Classification System with 19

6 Variation in the 8 of the Air stream Pulsed 21 (Simulated Feed, 1 Hertz, Slow Rise Active pulse)

7 Variation iActive Pulse shape (Simulated Feed, 1 Hertz, $50 \%$ of the Air stream Pulsed)

8 Variation in Frequency (Simulated Feed, Symmetric Active Pulse, $50 \%$ of the Air stream pulsed)

9 Variation in Active Pulse Shape (MSW Feed, 125 Hertz, $80 \%$ of the Air stream Pulsed)

10 Variation in Passive Pulse Shape (MSW Feed) 26

11 Summary of Maximum Efficiency Performance 27 (Phase III)

12 Summary of Efficient Separation Range Performance (Phase III)

13 Comparison of All Classifier Types Tall Height, Low Feed Rate, Phase III) 
I. AN INTRODUCTION TO AIR CLASSIFICATION FOR MUNICIPAL SOLID WASTE PROCESSING AND OBJECTIVES OF THIS RESEARCH REPORT

Research conducted at Duke University over the past 12 years has led to the development of a promising alternative method for processing municipal solid waste prior to energy production or resource recovery. Pulsed flow air classification is a laboratorytested method which successfully separates the combustible from the noncombustible components of MSW.

In 1986, Duke subcontracted to EG\&G Idaho to perform research in this area for the U.S. Department of Energy. This document represents the final report of that effort, and places this research in the context of all air classification research conducted at Duke University.

The goal of tris final report is to document the history of pulsed flow air classifier theory and design, while emphasizing test results which confirm success with MSW processing. The three objectives are:

- Summarize the pulsed-flow air classification theory developed at Duke University

- Document the equipment, procedures, test protocols, and results from research constructed at Duke University, with a focus on the combinations and classifier heights and feed rates which produce the best achievable separations, as well as a focus on the estimated cost of a complete classification system,

- Draw conclusions on the relative merits of selected air classifiers. 
The development of the theory and design of pulsed flow air classification is initially presented and experimental results are summarized. Pulsing and non-pulsing classifiers are discussed, and a complete system is designed and costed.

Appendix I provides a bibliography of relevant research. Appendix II summarizes the notation used in the report, and Appendix III presents the cost analysis conducted by EG\&G Idaho.

II. Development of Theory and Design: Historical Perspective

For the past 12 years air classifier research has been conducted at Duke University and the results are discussed here in chronological order. Early laboratory studies are seen to provide the basis for theoretical development of pulsed air classification.

1. Initial Laboratory studies of Smaller Scale Air classifiers Research concerning the effect of feed characteristics on air classifier effectiveness was initially addressed by Taub and Peirce (1983) using the zig zag classifier throat illustrated in Exhibit 1. Using a zig zag classifier with a 25 in $^{2}$ throat, 8 feet tall, in bench top studies, they found that varying the proportion of paper in the feed had a significant effect on the purity of the extract; i.e., the purity of the feed material reporting out the top of the classifier. Increasing paper content increased clogging in the throat and the feed material began behaving as clumps, not discrete particles. Non-combustible particles were observed 


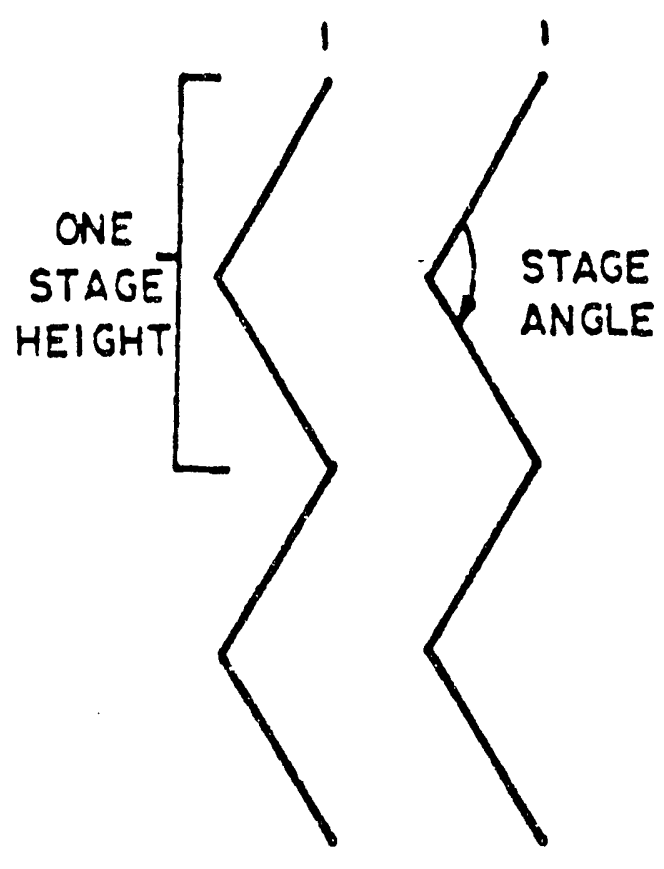

A: ZIG ZAG NON PULSED
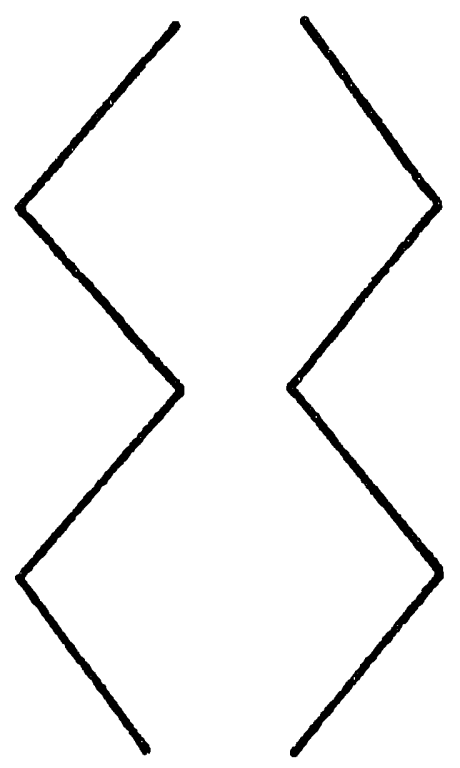

C: ZIG ZAG PASSIVE PULSED

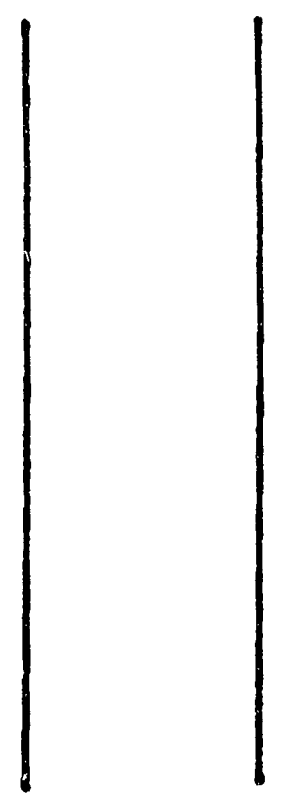
B: STRAIGHT NON PULSED OR ACTIVE PULSED (WITH LOUVRED VALVE)

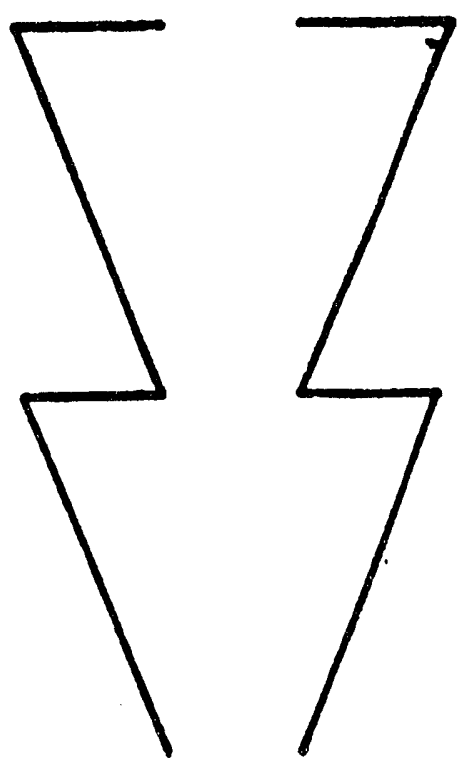

D: STACKED TRIANGLE PASSIVE PULSED

Exhibit 1. Air Classifier Throat Configurations 
trapped in the rising combustible material. The same effect was observed with a glass/paper feed mixture by saul (1981). Paper in the feed tended to trap small glass particles, carrying the glass with the combustible stream. Bartlett (1983) determined that $z$ ig zag air classifier separation becomes poor as MSW moisture content increases. Defining moisture content as the percent moisture in the absorbent material in the MSW, Bartlett found that performance was not affected in air dried to $86 \%$ moisture. From $86 \%$ to $230 \%$ moisture, absorbent combustibles (wet paper) recovery was reduced and above $300 \%$ moisture air classification was not feasible. Peirce, Vesilind and McNabb (1982) determined terminal velocities for irregularly shaped objects by measuring falling times. They determined that a separation of aluminum and plastic particles is impossible if the separation technique is based on aerodynamic differences because of terminal velocity overlap, discussed below. Peirce and Wittenberg (1984) studied the effect of zig zag air classifier configuration on the peak attainable efficiency. They changed classifier configuration by varying the stage angle and the number of stages and used different sized spheres as feed material. Peak efficiency was not observed to be influenced by classifier configuration within the ranges of these laboratory tests.

These early studies provided the basis for the theoretical development of pulsed air classification discussed below. Small laboratory systems are initially described; the results from larger scale laboratory tests are then presented. 
2. Theory Development and Subsequent Laboratory Investigations of Smaller systems

Typical air classifiers, such as the straight or zig zag shown in Exhibit 1, use a constant air stream to separate particles. with different terminal velocities. Terminal velocity is the falling velocity of a particle in a fluid or gas at which the particle falls at constant velocity and at which the sum of the Newtonian forces on the particle is zero. The terminal velocity of a sphere is:

$$
V_{t}=\left[\frac{4}{3} \frac{D g}{C_{D}} \frac{\rho_{p}-\rho_{f}}{\rho_{f}}\right]^{\frac{2}{2}}
$$

where $V_{t}=$ terminal velocity,

$$
\begin{aligned}
C_{D} & =\text { drag coefficient, } \\
\rho_{p} & =\text { particle density, } \\
\rho_{f} & =\text { fluid density, } \\
D & =\text { particle diameter, and } \\
g & =\text { gravitational constant. }
\end{aligned}
$$

The terminal velocity is a function of the particle diameter, the difference in density between the particle and fluid, and the drag coefficient. The separation capabilities of the typical air classifier depend on these three factors. 
The forces on a particle immersed in a fluid such as air are:

$$
F=F_{g}+F_{b}+F_{d}+F_{a r}
$$

$$
\text { where } \quad \begin{aligned}
F_{\mathrm{g}}= & \text { gravitational force, } \\
\mathrm{F}_{\mathrm{b}}= & \text { buoyancy force, } \\
\mathrm{f}_{\mathrm{d}}= & \text { drag force, and } \\
F_{\mathrm{ar}}= & \text { acceleration reaction force, resulting } \\
& \begin{array}{l}
\text { from the energy transfer required to } \\
\text { displace fluid as particles move through } \\
\text { it (Stessel 1983, Stessel and Peirce 1987). }
\end{array}
\end{aligned}
$$

At the point when a particle just begins to fall in a still fluid, the drag and acceleration reaction forces drop out. Equation (2) becomes:

$$
F_{g}+F_{b}=m_{p} a_{0}
$$

where: $m_{p}=$ mass of the particle, and

$$
\mathrm{a}_{\mathrm{p}}=\text { the particles initial acceleration }
$$

solving for $a_{0}$ :

$$
a_{0}=\frac{g\left(\rho_{p}-p_{t}\right)}{\rho_{p}}
$$

Thus, as the particles just begin to fall, their acceleration is a function of density only. This is crucial to the success of pulsed flow air classification.

To develop this concept further, consider four spheres whose 
falling velocities from rest are shown in Exhibit 2 . Spheres $A$ and $B$ are more dense, while $C$ and $D$ are less dense than some reference value. However, their diameters are such that spheres $A$ and $C$ exhibit higher terminal velocities than $B$ and $D$. A standard classifier can only separate spheres $A$ and $C$ from $B$ and $D$, but density separation would separate spheres $A$ and $B$ from $C$ and $D$. Exhibit 2 indicates how this can be achieved. Until $t_{1}$ seconds after release, the velocity of $B$ is higher than sphere $C_{i}$ thus, B falls farther. Between $t_{1}$ and $t_{2}, B$ still falls farther than $C$ (Area $I=$ Area II) but $c$ exhibits a higher velocity than B. At $t_{2}$, the particles meet again and C overtakes B. If density separation is desired, the particles must be kept in a behavior regimen somewhere to the left of $t_{2}$ in Exhibit 2 . The particles must be accelerated and stopped repeatedly so that the time of free fall is well below $t_{2}$. Pulsed air classification pulses the air flow in order to achieve this effect.

Two types of pulsing action have been developed at Duke: passive and active. In passive pulsing air classifiers (two designs of which are shown in Exhibit 1), the air flow varies with position in the classifier, thus the air velocity varies with distance. This pulse is produced by constructing the walls of the classifier to make the throat alternatively wide and narrow. Active pulsing air classifiers vary the flow with a shutter or gate valve which repetitively constricts and frees the air flow creating a periodic time dependent air flow.

Peirce, stessel and Wolf (1986) compared the performance of smaller scale (approximately 25 inches square flow channels) 


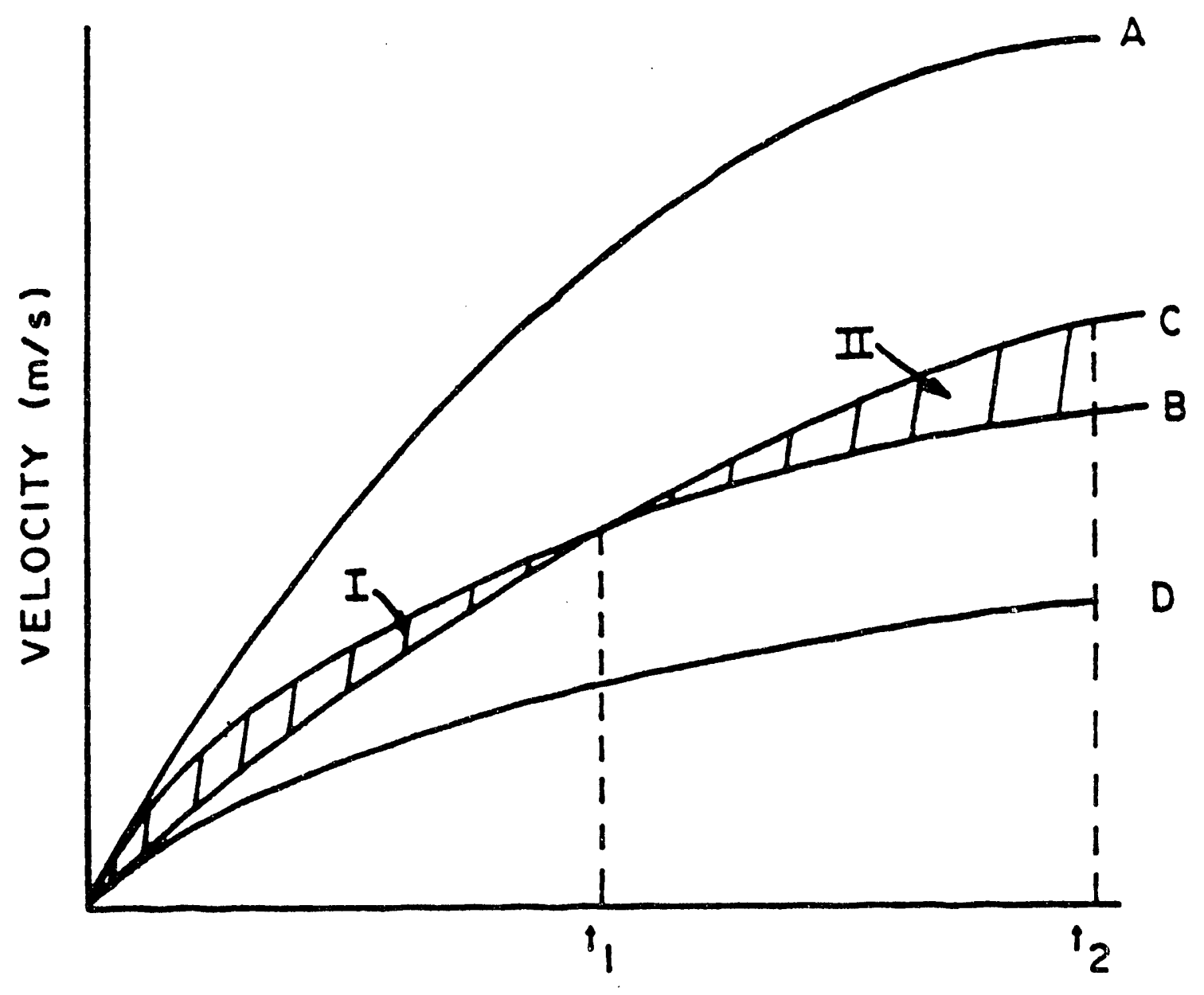

TIME ( sec)

Exhibit 2. Spheres Falling from Rest 
passive pulsing to nonpulsing air classifiers. They documented the flow regimes in zag zag and stacked triangle passive pulsing classifiers, determined that distinct regions of high and low velocities are created by the wall geometries, and showed that these classifiers are passive pulse air classifiers. The passive pulsing classifiers exhibited higher maximum peak efficiencies and larger operating ranges than straight and zig zag classifiers. Jackson, Stessel and Peirce (1988) presented a mathematical model used to study the behavior of spheres in idealized passive pulsing air classifiers. The model predicts that passive pulsing can reverse falling order for spheres like $B$ and $C$ but not separate them on a density basis; i.e., $B$ can be made to fall faster than $C$ but $B$ cannot be lifted while $C$ falls. Crowe and Peirce (1988) documented flow regimes showing that passive pulsing occurs in larger scale, approximately one foot square, zag zag and stacked triangle classifiers. The larger scale passive pulsing classifiers performed better than nonpulsing straight and zig zag classifiers on plastic/aluminum platelet and simulated MSW feeds.

Stessel and Peirce (1983, 1985, 1986, 1987) developed a theoretical model based on a fluid mechanical force balance to describe the behavior of spheres in an actively pulsed air flow. Both the theoretical model and experimentation with small scale air classifiers indicate that active pulsing air classifiers can achieve density separation of spheres with reversed aerodynamic characteristics like sphere $B$ and $C$ mentioned earlier. Laboratory experimentation showed that best separation occurred with a long attack waveform, as the majority of the pulse period is devoted to a slow rise of the air velocity followed by a quick drop, with straight parallel air classifier walls (Stessel and Peirce 1983, 1985). Pulse frequencies 
near 1 hertz and pulsing of the entire air flow lead to better separation (Stessel and Peirce 1986, 1987). Laboratory batch-feed and continuous feed comparisons of the small scale active pulse air classifier to other types of classifiers showed clear superiority for active pulsing. Everett and Peirce improved the theoretical model to allow spheres to be injected at any time during the air pulse. Their results suggest that synchronizing feed input with pulse time may increase classifier efficiency (Everett and Peirce, 1989).

The passive and active pulsing models developed by Peirce, St ssel, Jackson and Everett do not allow for particle-particle or particle-wall interaction and are limited to modelling the behavior of spheres. The extremely heterogeneous nature of $M S W$ and the difficulties encountered modelling particle-particle and particle-wall interactions make experimental testing of the pulsing classifiers very important, especially tests with simulated and real MsW feed material.

III. Laboratory Equipment, Procedures, and Test Protocol

A complete air classifier system was constructed to study the separation of the combustible from the non-combustible components of MSW. Detailed procedures and a parametric test protocol were developed. This equipment, procedures, and test protocol is discussed below.

1. Equipment

The air classifier system used in this project was designed and constructed at Duke University. The shapes of the classifiers used are 
shown in Exhibit 1. The throat widths were:

- $0.30 \mathrm{~m} \mathrm{(12} \mathrm{in)} \mathrm{for} \mathrm{the} \mathrm{straight} \mathrm{and} \mathrm{active} \mathrm{pulse,}$

- $0.33 \mathrm{~m}$ (13 in) for the $z i g z a g$, and

- $0.25 \mathrm{~m}(10 \mathrm{in})$ at the narrowest point and $1.14 \mathrm{~m}(45 \mathrm{in})$ at the widest point for the zag zag and stacked triangle.

In the cases of the zig zag, zag zag and stacked triangles, each stage is $0.76 \mathrm{~m}(2.5 \mathrm{ft})$ tall, and classifiers of 3 and 6 stages were studied. Thus the total classifier length ranged from $2.3 \mathrm{~m}(7.5 \mathrm{ft})$ to $4.6 \mathrm{~m}$ (15 ft). The thickness of each throat was .30m (12 in). The air speed was varied by adjusting a sliding gate valve between the fan and the connecting hosing illustrated in Exhibit 3. Particles were fed into the second or third stage from the bottom with 3 or 6 stage classifiers respectively. The entire system is shown in Exhibit 3. Two components were modified for certain tests. When non-pulsing or passive pulsing air classifiers were tested the induction fan and sliding gate valve were moved to the far end of the settling chamber $H$. In this configuration air was pulled through the air classifier system. When feed rate variations were tested, conveyor $B$ was removed and staging table A placed adjacent to surge in C. This allowed for higher feedrates.

The air velocity was measured using a TSI 1053-1 hot film anemometry system, consisting of a $1053 \mathrm{~B}$ nonlinearized anemometer and a 1051-1 power supply and analog readout. The probe used a 1210-60 W heavy-gauge film sensor and associated mounting hardware. The probe was screwed into the classifier wall at a port located along the center line of the classifier and was inserted into the throat center. The 


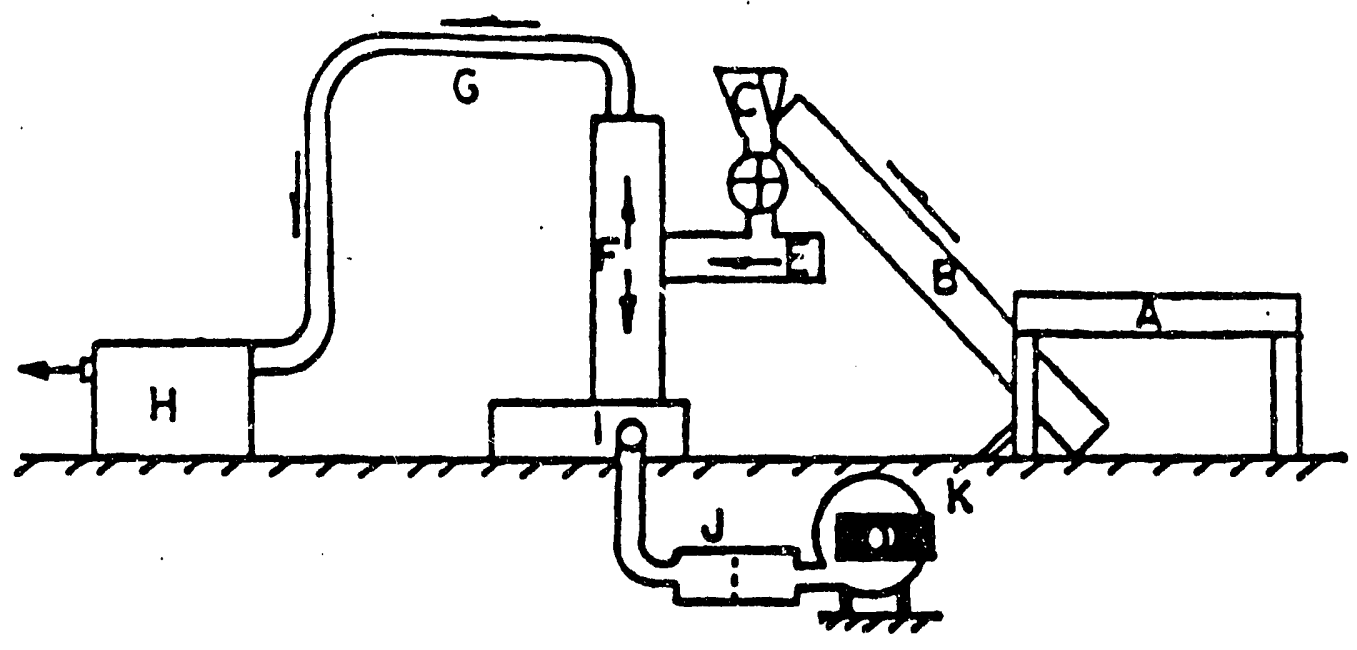

- A - Sioging Table

B. Conveyor

$C$ - Surge Bin

D. Alrlock Volve

E * Mini. Conveyor

$F$ - Clussifler Throat

$G$ = Duci Work

$H$ - Exiraci Seftling Chomber

1 - Rejecs Sefrling Chamber

$J$ - Pulsing Valve

$K$ - Fon with Sliding Gate Volve 
voltage drop across the sensor was read by a voltmeter and converted to the corresponding velocity as calibrated by the vendor. The conversion incorporated a correction for the ambient temperature. Time dependent air flow pulses were recorded by linking the anemometer to a Vector personal computer via a 12-bit analog input module. Equivalent turbulence for the system was $0.03 \%$; hence, the $95 \%$ confidence interval was $\pm 0.06 \%$.

Periodic pulses were created using a louvered valve designed at Duke University. Three plates affixed to three axles perpendicular to air flow were moved by a pneumatic piston to alternately block and unblock the air flow throughout the valve. By adjusting the expansion and contraction of the piston different pulses were created. BY adjusting the plates to block more or less of the air flow the percent of the air flow pulsed was changed. With the plates set to close completely, blocking all of the air flow, $100 \%$ of the air flow was pulsed. If the plates remained fixed in the open position $0 \%$ of the air flow was pulsed. The valve was capable of producing waves with frequencies between selected ranges:

- for symmetric pulses, frequencies from

$0.5 \mathrm{~Hz}$ to $2.5 \mathrm{~Hz}$, and

- for asymmetric pulses, frequencies

from $0.5 \mathrm{~Hz}$ to $1.5 \mathrm{~Hz}$.

Furthermore, due to leaks, the valve developed at Duke was not able to pulse more than $80 \%$ of the air flow. The pulses produced were periodic; air flow velocity increased from a minimum to a maximum value then decreased back to the minimum. By adjusting the rates of expansion and contraction of the valve pistons the percentage of the pulse period devoted to rising air flow was changed. Quick 
rise, slow rise and symmetric pulse shapes were used in this research corresponding to pulses with much less than $50 \%$, much more than 50\%, and 50\% of the pulse period devoted to increasing air velocity, respectively. A typical saw-tooth symmetric air pulse is shown in Exhibit 4. Piecewise linear regression techniques were applied to data obtained prior to maximum air velocity and, separately, to flow data obtained after maximum air velocity was attained. These results are also shown in Exhibit 4 as straight line segments through the actual data plot.

Feed samples consisted of shredded simulated MSW made in the lab and real shredded MSW obtained from the field. These samples were tested in a four-phase test prot.ocol discussed in detail at the end of the chapter. Phase I tests used simulated MSW with $65 \%$ newspaper, $20 \%$ water, $5 \%$ steel, $5 \%$ plastic, and $50 \%$ aluminum. The sample was shredded to < 1 inch with available laboratory equipment except one half of the newspaper fraction which was cut into $\leq 3$ inch squares to better approximate field conditions. Phase II tests used MSW obtained from the Charleston, South Carolina Waste Management Authority shredded to $<4$ inches at the Charlotte facility. Samples were cut and quartered to obtain approximately $3 \mathrm{~kg}$ random samples for testing. Because of the long time required to conduct each test, MSW was collected from the charlotte facility at two different times, once for passive pulse testing and once for active pulse testing. MSW simply does not remain biologically stable long enough to permit a single example to suffice to test both classifiers. Thus comparisons of the performance of passive 


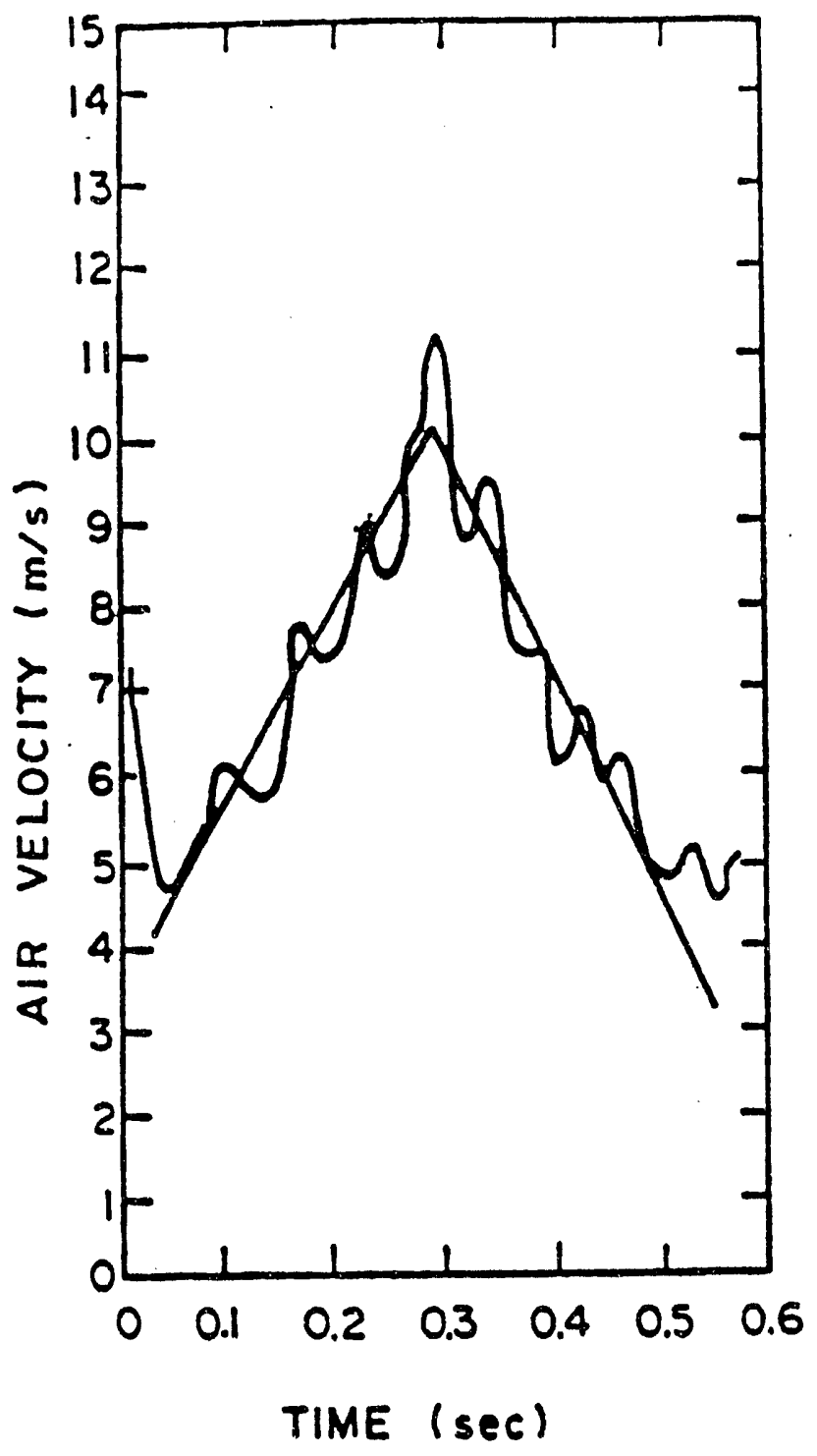

Exhibit 4. Air Velocity Graph: Symmetric Wave Form 
and active pulsing air classifiers in Phase II are somewhat difficult to make. Phase III tests used simulated MSW with $55 \%$ newspaper, $20 \%$ water, $10 \%$ plastic, and $5.3 \%$ aluminum shredded to $<$ ? inch with available laboratory equipment with a focus on feed rate and classifier height. Phase IV tests used simulated MSW to examine the usefulness of a screw conveyor system and a cyclone with the pulsed flow classifiers.

\section{Procedures.}

Particles were injected into the classifier system in a fourphase test protocol. In each phase of testing procedures, classifier efficiency was defined as (Peirce, Vesilind, and Stessel 1983):

$$
E=\sqrt{\frac{X_{e}}{X_{0}} \cdot \frac{Y_{r}}{Y_{0}}} * 100
$$

where: $\quad E$ = Efficiency $(\xi)$

$$
\begin{aligned}
x_{e}= & \begin{array}{l}
\text { Mass of combustibles exiting as lights } \\
\text { through top of classifier }(\mathrm{gm})
\end{array} \\
x_{0}= & \text { Mass of combustibles input to classifier (gm) } \\
y_{r}= & \begin{array}{l}
\text { Mass of non-combustibles existing as heavies } \\
\text { through bottom of classifier (gm) }
\end{array} \\
y_{0}= & \text { Mass of non-combustibles input to classifier (gm) }
\end{aligned}
$$

This $\mathrm{E}$ value is used in this report to represent the efficiency of a particular classifier in separating a certain sample at a 
specific mean rising air velocity.

The separation efficiencies and corresponding air velocities for each classifier configuration are discussed later in this report. Data plots permit the observation of two aspects of separator performance:

- The comparative peak efficiencies attained by each classifier, and

- The velocity ranges over which each classifier is capable of exceeding a particular separation efficiency, called the efficient separation range. In the work presented here, when this performance parameter is used, a separation efficiency of $90 \%$ is chosen.

Variations in feed characteristics and feed rates can drastically affect the air velocities within the classifier throats and thus drastically affect air classifier performance. Thus, classifiers performing efficiently over a wide range of air velocities are preferred to classifiers which do not operate well over a wide range of air velocities. Hence the efficient separation range becomes critical when comparing the performance of different classifiers.

\section{Test Protocol}

A four-phase test protocol was followed. In Phases I and II three stage, 2.3 meter $(7.5 \mathrm{ft})$ classifiers were tested. In Phase I the active pulse air classifier was tested with simulated shredded MSW. Variations in the percentage of the air flow pulsed, the pulse shape, and the pulse frequency were tested laying the groundwork for tests with shredded MSW. In Phase II both active 
and passive pulse classifiers were tested with shredded MSW. Three pulse shapes were tested: symmetric, quick rise, and slow rise. Zag Zag and stacked triangle passive pulse classifiers were also tested. Because of time restrictions in the research tasks, two different MSW samples were collected from Charleston, South Carolina; thus it is difficult to make direct comparisons between active and passive pulsing classifiers with real feed. During Phase II, it was found that the system would not permit complete analysis of all mean rising air velocities for all classifiers. The fan simply was not capable of producing sufficiently high velocities in the classifier thrusts. Phase III was designed to address this limitation. In Phase III zig zag non-pulse, stacked triangle passive pulse and 0.7 hertz, long attack time, $80 \%$ air flow pulsed active pulse were tested over a complete range of air velocities with simulated shredded MSW for two feed rates and classifier lengths. Feed rates of $3.6 \mathrm{~kg} / \mathrm{minute}(0.25 \mathrm{~s} l \mathrm{ug} / \mathrm{minute})$ and $7.2 \mathrm{~kg} / \mathrm{minute}(0.50 \mathrm{slug} / \mathrm{minute})$ and heights of 2.3 meters $(7.5$ $\mathrm{ft})$ and 4.6 meters $(15 \mathrm{ft})$ were used corresponding to 3 and 6 stages, respectively. This allowed for comparison of the three types of classifier under four conditions. In Phases I, II, and III, non pulsing classifiers were tested for benchmark comparisons. In Phase IV of the research, a complete passive air classification system was constructed with a screw conveyor and a cyclone. As seen in Exhibit 5, this system consisted of: a screw conveyor for continuous feed, a stacked triangle passive classifier throat, with dimensions identical to the throat studied in Phases I, II and III, and a cyclone to collect the particles exiting the top of the classifier. 


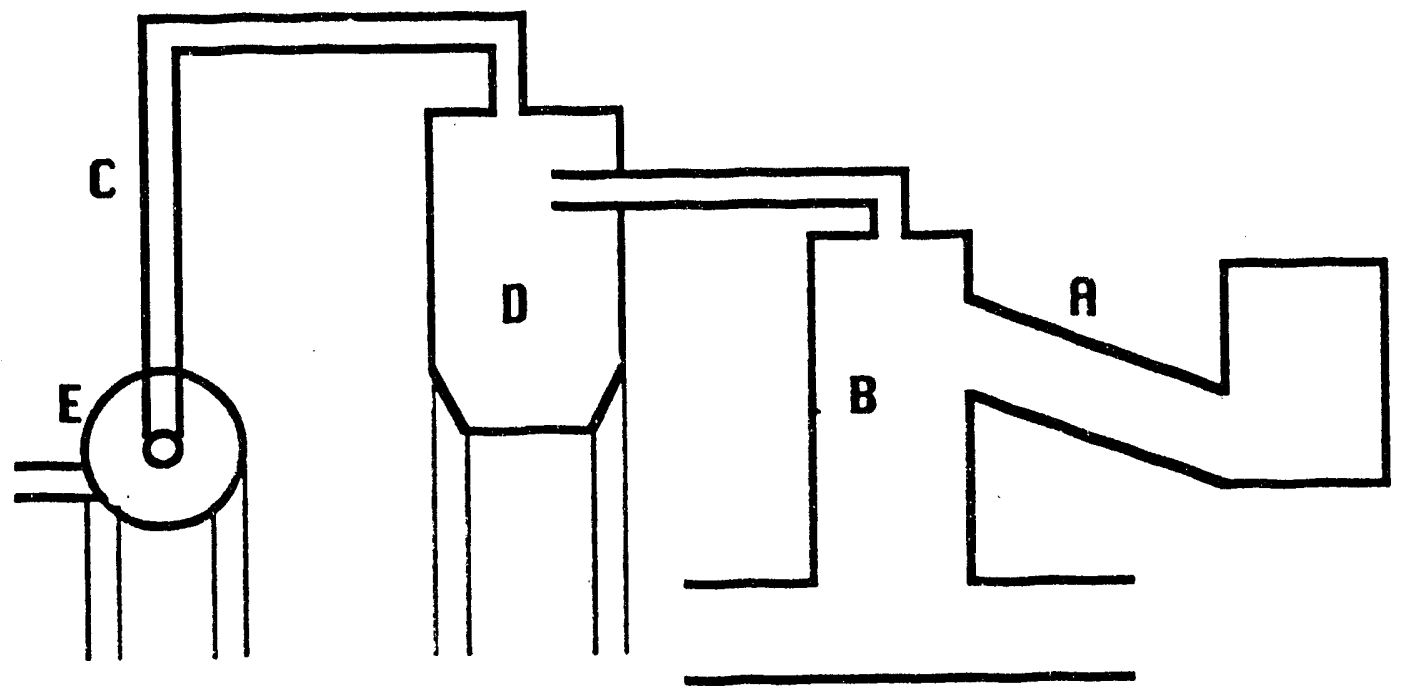

$A=$ SCREW CONUEYOR

$B=$ STACKED TRIANGLE CLASSIFIER

$\mathrm{C}=$ DUCTS

D= CYCLONE

$\mathbf{E}=\mathbf{F} \boldsymbol{A N}$

Exhibit 5. Passive Air Classifier System With Cyclone 
IV. Results and Discussion

During the construction and shakedown of the test equipment, it was determined that data collection was arduous, labor intensive and expensive. For example, the calculation of the separation efficiency for a single sample was particularly time consuming. Each sample typically consisted of thousands of pieces of paper, plastic, aluminum and what not. Each piece of plastic, aluminum, paper and the whole range of real components of MSW had to be hand selected and hand sorted; each sample contained many thousands of individual pieces. Attempts were made to determine if a single sample test at each air velocity would give reproducable results. For example, three tests were performed on a zig zag air classifier at one air velocity. The separation efficiencies of the three replicates are $89.1 \%, 91.4 \%$, and $92.0 \%$. The standard deviation is calculated as $1.5 \%$ and the $90 \%$ confidence interval is E士 $2.6 \%$. Thus it was determined that a single sample at each air velocity was acceptable when results over a range of air velocities were consistent.

The results of Phase I of the Test Protocol are summarized in Exhibits 6, 7, and 8. Note that data points are connected for all test results of the non-pulsing classifiers to facilitate benchmark comparisons with the results of the pulsing classifiers. In Exhibit 6 increasing the percentage of the air flow pulsed increases air classifier efficiency. The results shown in Exhibit 7 and 8 are not as clear. Exhibit 7 shows the symmetric pulse classifier outperforming the other classifiers (Stessel and Peirce 1983, 1985, 1986, 1987 and Everett and Peirce 1989). In Exhibit 8 


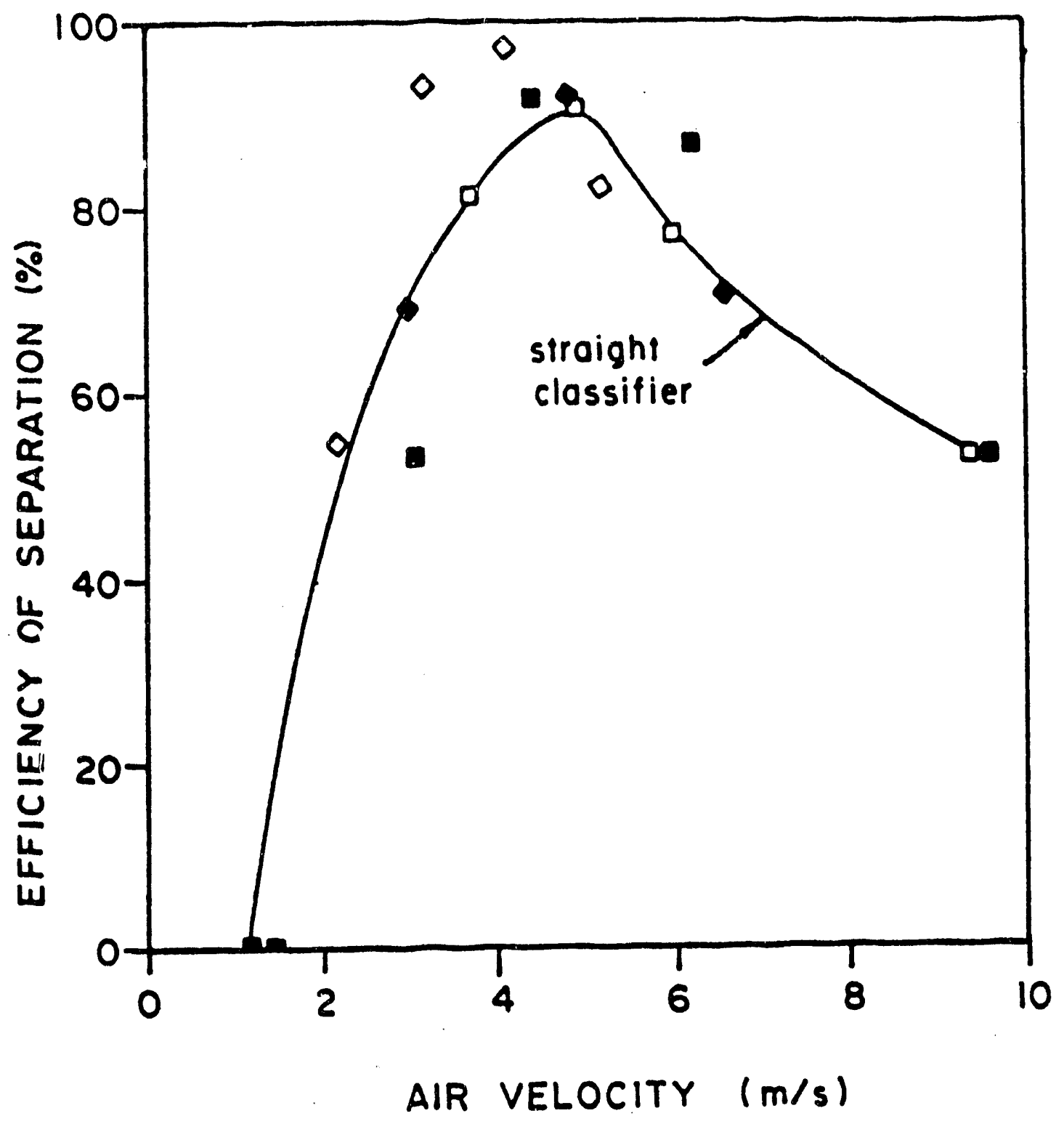

-

- $20 \%$ of oir siream pulsed, active pulsing

- $50 \%$ of air siream pulsed,octive pulsing

- $80 \%$ of air siream pulsed, octive pulsing

Exhibit 6. Variation in the $z$ of the Air stream Pulsed (Simulated Feed, I hertz, slow rise active pulse) 


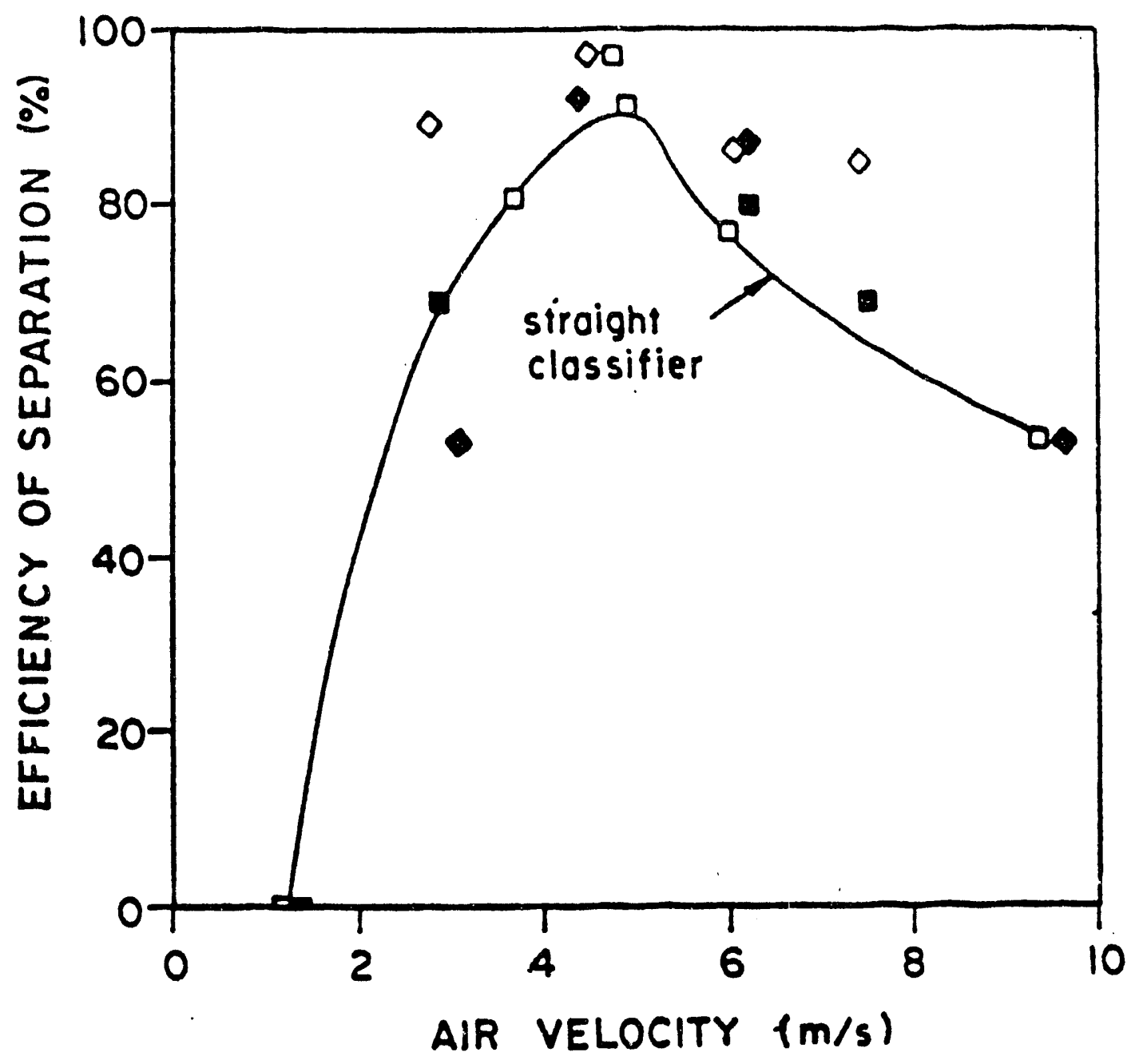

-o- straight, non pulsing

- slow rise pulse, octive pulsing

- quick rise pulse, active pulsing

- symetric pulse, active pulsing

Exhibit 7. Variation in Active Pulse shape (Simulated Feed, 1 Hertz, $50 \%$ of the Air stream Pulsed 


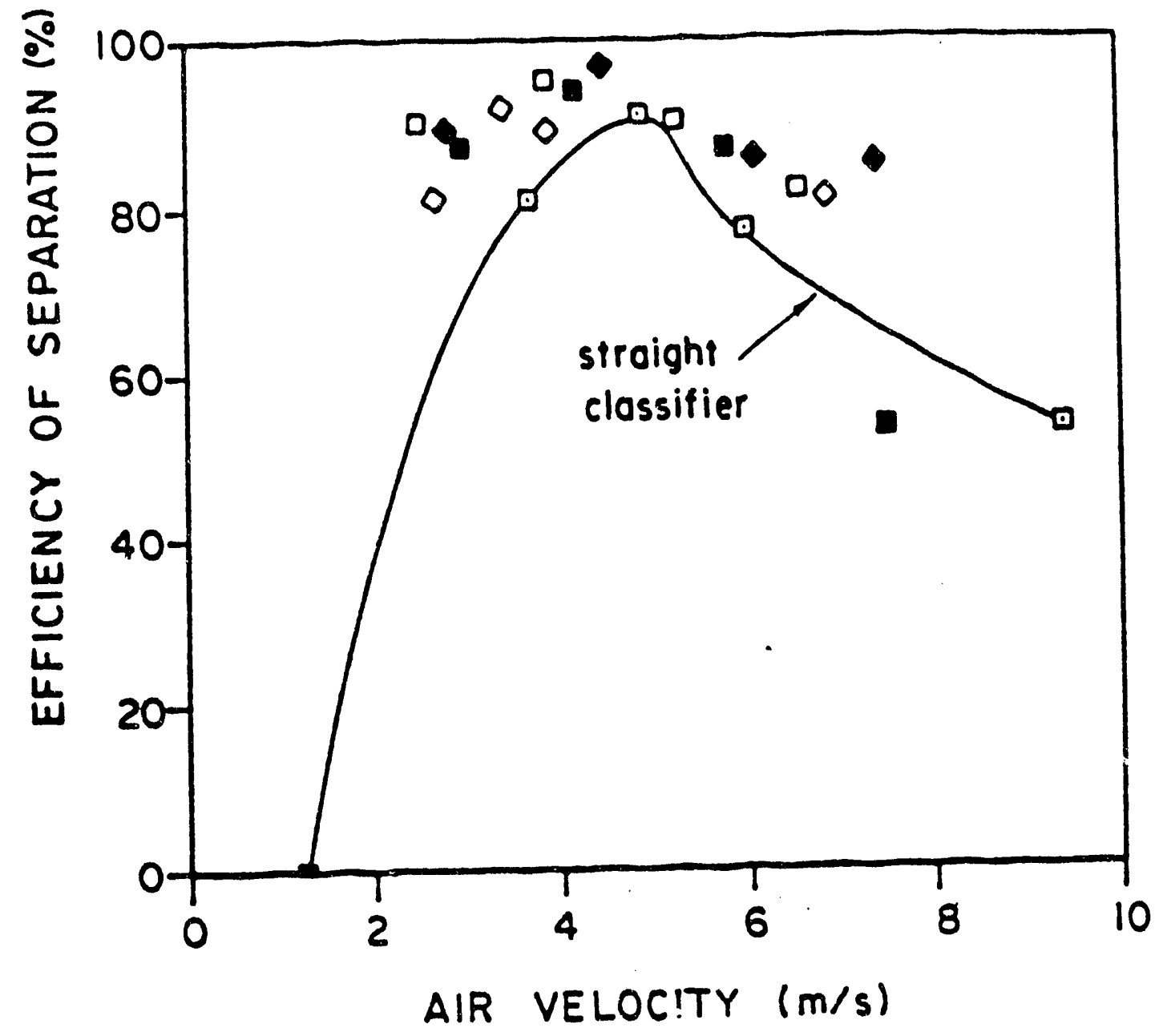

- siraight, non pulsing
I hertz frequency, octive pulsing
0.5 heriz frequency, octive pulsing
$0 \quad 1.5$ heriz frequency, octive pulsing
2.45 heriz frequency, octive pulsing

Exhibit 8. Variation in Frequency (simulated Feed, Symmetric Active Pulse, 50\% of the Air Stream Pulsed 
no trend is discernible. However, in all three graphs the activepulsing classifiers outperform the non pulse straight classifier.

Exhibits 9 and 10 show the results of Phase II experimentation. In Exhibit 9 the efficiuncy performance of three active pulse and a zig zag classifier are shown. Again, the symmetric pulse performance seems to be superior, contrary to theory. However, as theory to date has been concerned with modelling spheres, this may not be too surprising; this result is consistent with the laboratory studies conducted with simulated shredded MSW.

Exhibit 10 shows results from testing $z a g$ zag and stacked triangle passive pulse classifiers along with straight and zig zag non pulsing classifiers. The results present a mixed picture. It is clear that the straight classifier performs most poorly, but the complete efficiency curve was not available for the zig zag (because of the unexpected flow limitations of the fan), and it is not possible to compare its operating range with the passive pulsing classifiers. Depending on efficiencies developed at greater air velocities the zig zag could be superior or inferior to the zag $z 2 g$ and stacked triangle air classifiers. The availability of a more powerful fan or perhaps smaller shredded MSW particles would have allowed those efficiencies to be mapped. Phase III of the test protocol was designed to address this problem.

The results of Phase III are shown in Exhibits 11,12 and 13 . Because complete efficiency curves over a complete air velocity range could be mapped for each classifier, comparisons can be conducted using both peak efficiencies and efficient separation ranges of greater than $90 \%$ efficiency. Exhibit 11 shows that all 


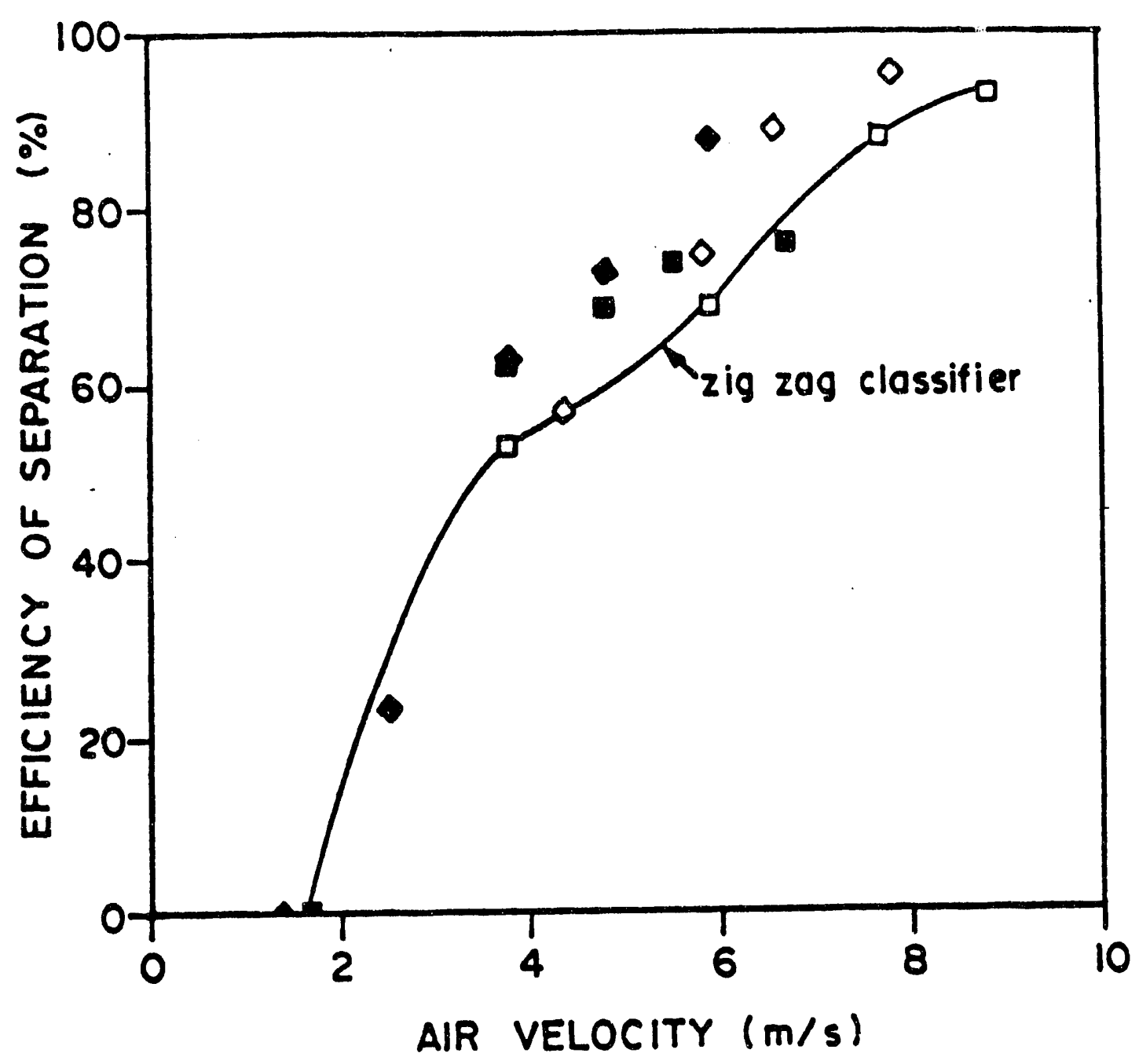

- zig zag, non pulsing

- quick rise pulse, ocrive pulsing

- slow rise pulse, octive pulsing

- symetric pulse. octive pulsing

Exhibit 9. Variation in Active Pulse Shape (MSW Feed, 1 Hertz, $80 \%$ of the Air Stream Pulsed) 


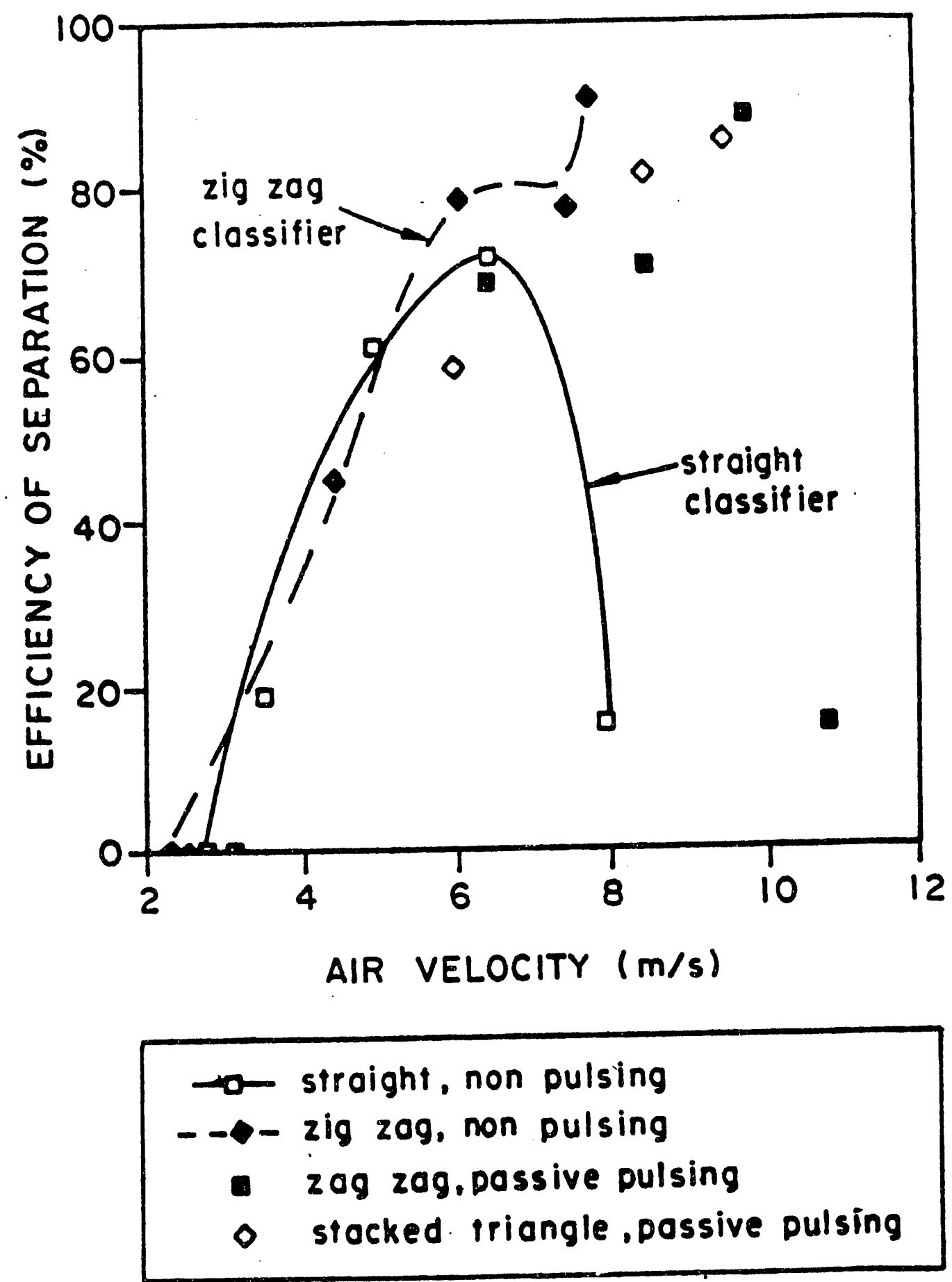

Exhibit 10. Variation in Passive Pulse Shape (MSW Feed) 
Exhibit 11. Summary of Maximum Efficiency Performance (Phase III)

\begin{tabular}{|c|c|c|c|c|}
\hline \multirow[b]{3}{*}{$\begin{array}{c}\text { Classifier } \\
\text { (1) }\end{array}$} & \multicolumn{4}{|c|}{ Maximum Efficiency } \\
\hline & \multicolumn{2}{|c|}{ Short Classifier } & \multicolumn{2}{|c|}{ Tall classifier } \\
\hline & $\begin{array}{l}\text { High Feed } \\
\text { Rate } \\
\text { (2) }\end{array}$ & $\begin{array}{l}\text { Low Feed } \\
\text { Rate } \\
\text { (3) }\end{array}$ & $\begin{array}{l}\text { High Feed } \\
\text { Rate } \\
\text { (4) }\end{array}$ & $\begin{array}{l}\text { Low Feed } \\
\text { Rate } \\
\text { (5) }\end{array}$ \\
\hline $\begin{array}{l}\text { Zig Zag } \\
\text { Non Pulse }\end{array}$ & 94 & 95 & 96 & 99 \\
\hline $\begin{array}{l}\text { Louvred } \\
\text { Valve } \\
\text { Active } \\
\text { Pulse }\end{array}$ & NA & NA & 99 & 97 \\
\hline $\begin{array}{l}\text { stacked } \\
\text { Triangle } \\
\text { Passive } \\
\text { Pulse }\end{array}$ & 96 & 99 & 97 & 99 \\
\hline
\end{tabular}

NA = Not available due to time constraints. 


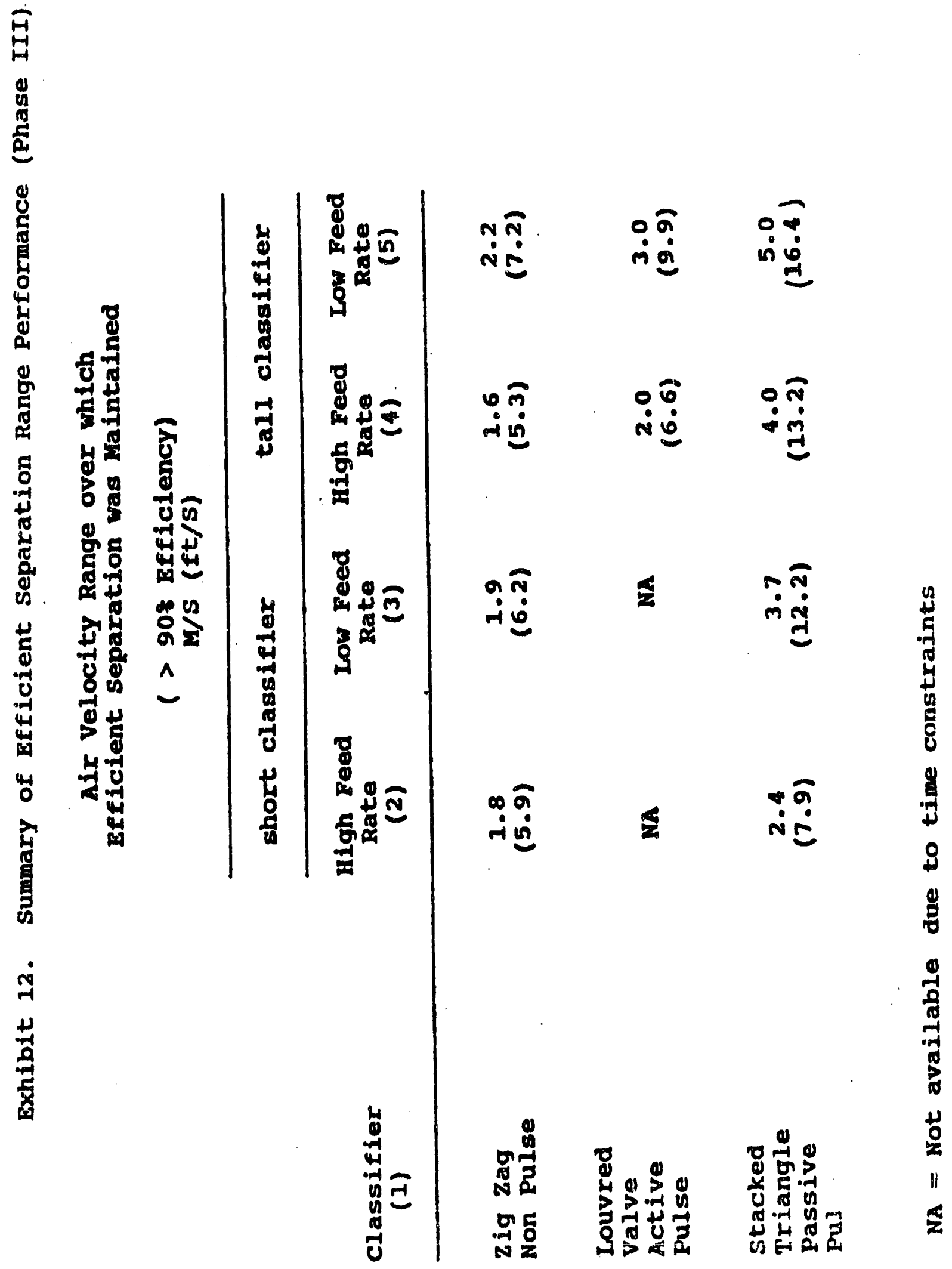




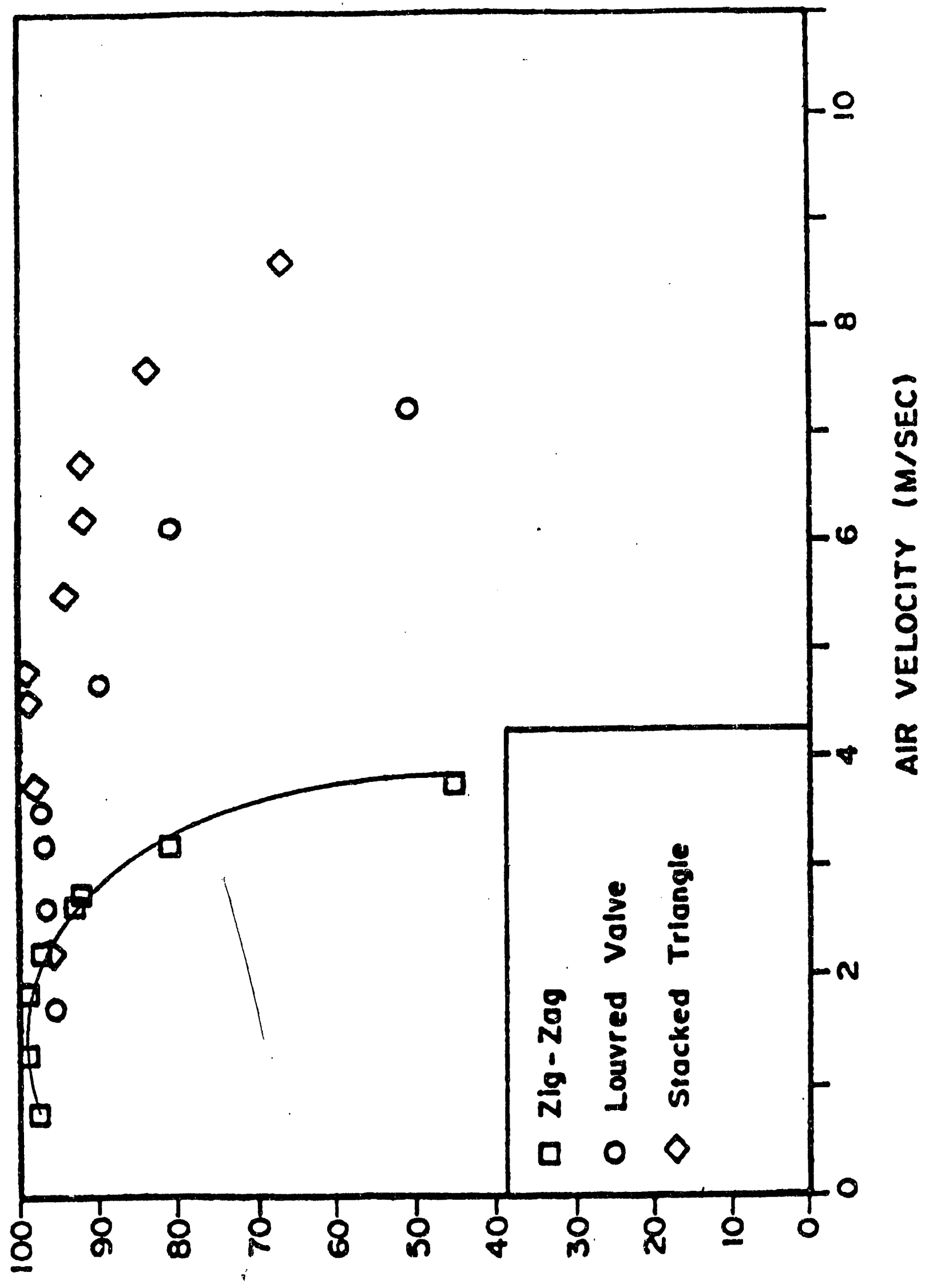

Exhibit 13. Comparison of All classifier Types (Tall Height, Low Feed Rate, Phase III) 
of the classifiers were able to reach high efficiencies with the simulated shredded MSW used. The stacked triangle achieved the highest efficiency for all variations of feed rate and classifier height except when it was tested at the tall height/high feed rate combination. The active pulse classifier excelled at that height and feed rate. Because all of the classifiers achieved high peak efficiencies, and because no statistical significance can be subscribed to peak efficiency differences, it is important to examine their efficient separation ranges. These are shown in Exhibit 12 and demonstrated graphically for one height and feed rate in Exhibit 13. The trend is clear. The stacked triangle passive pulse outperforms the louvered valve (active pulse) which in turn outperforms the zig zag (non pulse). The efficient separation ranges of the passive and active pulse classifiers are 2.0 and 1.3 times greater than that of the nonpulsing classifier, respectively.

Recall that in earlier research smaller active pulsing classifiers appeared to outperform passive pulsing classifiers. This might be due to several factors. Different and more effective pulses may have been achieved in smaller systems. The smaller size of the classifiers may have effected the results because of different particle-particle interaction patterns. It appears that increasing height and decreasing feed rate increase classifier efficient separation range. This result is further discussed in a paper by Everett and Peirce (1989b).

Earlier research projects at Duke University in the air 
classification of MSW concluded that efficiency versus air velocity curves are parabolic (steșsel and Peirce, 1986 and Peirce and Wittenberg, 1984). Consequently, these initial studies fit parabolic curves to data and calculated peak efficiencies and overall operation ranges from the parabolic fits. However, the research presented here shows that these curves often exhibit plateaus and are not parabolic. Consider the stacked triangle data shown in Exhibit 13. Velocities of 6.2 and $6.7 \mathrm{M} / \mathrm{s}(20.3$ and 22.0 $\mathrm{ft} / \mathrm{s}$ ) both result in separation efficiencies of $92.0 \%$. This can be explained. At each air velocity almost all (99) of the combustible fraction was extracted out the top of the classifier and $85 \%$ of the noncombustible fraction was rejected through the bottom of the classifier. The ability of the classifier to separate combustible from non-combustible particles thus did not change between 6.2 and $6.7 \mathrm{~m} / \mathrm{s}(20.3$ and $22.0 \mathrm{ft} / \mathrm{s})$. Depending on the feed material many plateaus can be possible. For this reason parabolic curves are not fitted to the data presented here; rather, the peak efficiencies and efficient separation ranges are summarized in Exhibits 11 and 12.

Phase IV of the research focused on the design, construction and cost analysis of a complete air classifier system illustrated in Exhibit 5. The system was designed and constructed by personnel at Duke University; the cost analysis was conducted by personnel at EG\&G Idaho. 
The system was constructed and preliminarily tested with simulated MSW. All components performed as expected:

- the screen conveyor fed the MSW into the throat of the classifier: occasional clogging was observed in the conveyor as moisture increased in the MSW

- the classifier performed as predicted in earlier test phases as discussed above in terms of air flow characteristics and separation efficiencies.

the cyclone was able to be $100 \%$ effective in capturing the particles exiting the top of the classifier.

Thus a system was successfully constructed and seems to perform as anticipated with a capacity of 1.5 tons of MSW per day.

The cost analysis constructed by EG\&G Idaho, as presented in Appendix III, indicates an equipment cost of $\$ 44,096$. The total cost of $\$ 90,000$ in 1988 . dollars include directs, indirects, engineering design, inspection, project administration and contingency. Construction management costs are excluded. 
v. CONCLUSIONS OF THIS RESEARCH

1. The long history of air classifier research and development at Duke University led to a new theory and designs which have wide application. Early laboratory studies point to the importance of feed composition, including paper-to-glass ratios and moisture content. subsequent theoretical developments and laboratory studies of small scale classifiers demonstrate the existence of pulses in carefully designed throats. This same work also indicated the superiority of pulsing classifiers over non-pulsing classifiers. Mathematical models of spherical particles have been developed to explore the isolated phenomena of active and passive pulsing. These theoretical developments and empirical results lead to the most recent studies of large pulsed air classifiers also discussed in this paper.

2. The active pulsed air classifier valve constructed at Duke University is useful to study pulsed air flows but simited in the range of parameters which can be tested:

- erequency between $0.5 \mathrm{hz}$ and $2.5 \mathrm{hz}$ for symmetric pulse shapes,

- frequency between $0.5 \mathrm{hz}$ and $1.5 \mathrm{hz}$ for asymetric pulse shapes, and

- percent air pulsed between $20 \%$ and $80 \%$. 
3. The large scale active pulsing classifiers perform at higher efficiencies over greater air velocity ranges than the straight or zig zag non pulsing classifiers with both real and simulated shreaded MSW feeds.

4. The performance of the active pulsing system appears to be independent of frequency within the range tested; 0.5 to $2.5 \mathrm{hz}$.

5. Pulse shape in active pulsing air classifiers is important to classifier performance. Quick rise and symmetrically pulsed air flows appear to separate particles more effectively than slow rise pulsed air flows. This is indicated in the results of 6 separate tests. Each of three pulse shapes was tested under two different conditions: pulse frequency of $1 \mathrm{hz}, 50 \%$ air flow pulsed, simulated MSW; pulse frequency of $1 \mathrm{hz}, 80 \mathrm{z}$ air flow pulsed, real shredded MSW.

6. The portion of the air flow which is actively pulsed is important to air classifier performance. By blocking more or less of the air flow, the percent of the air flow pulsed can be changed. As the percentage of air which is periodically blocked, and thus pulsed, increases from 208 to 508 to $80 \%$, air classifier performance improves. 
7. When non-pulsing, passive pulsing and active pulsing classifiers were tested with the same simulated shredded MSW the results obtained are very clear: the passive pulsing classifiers outperform the active pulsing classifiers which in turn outperform the nonpulsing classifiers. However, these systems have not been optimized; active and passive pulsing configurations not yet studied could lead to a better understanding of the pulsing systems.

8. Results are not complete with real shredded MSW for passive pulsing classifiers. The fan used in the laboratory was not powerful enough to allow complete mapping of the efficiency curves at high air velocities. Only large 4 inch MSW was available and the fan in the system simply could not lift all heavier particles. However, the stacked triangle and zig zag passive pulsing air classifiers clearly performed better than the straight non-pulsing classifier over the air velocities achieved in the laboratory. Comparisons between these passive pulsing and the zig zag non-pulsing classifiers with real MSW cannot be made with confidence at high air velocities due to the limitations of the fan.

9. Results obtained from tests with simulated shredded MSW and shredded MSW did not contradict, each other. It appears that simulated shredded MSW can be used in tests conducted to aid in the design of shredded MSW air classifiers. Expensive, time consuming testing with actual MSW appears to be useful only in tha field as systems are fine-tuned to separate the combustible and the non-combustible portions of MSW from particular municipalities. 
10. The tall passive-pulse stacked-triangle air classifier is clearly superior to the tall active-pulse and tall non-pulse classifiers. The tall passive-pulse classifier achieved the broadest efficient separation range for each combination of height/number of stages and feed rates tested, achieving an efficient separation range over twice that of the maximum range attained by the non-pulse classifier and 1.7 that of the active-pulse classifier.

11. Increasing the feed rate decreased the efficient separation range for the classifiers tested. Further, increasing the classifier height and stage number tends to increase the efficient separation range for the classifiers tested.

12. A complete continously fed 1.5 tons $M S W / h r$, passive pulsing air classification system with a screw conveyor and a cyclone can be constructed for a total cost of $\$ 90,000$, of which $\$ 44,100$ is for equipment. This system could provide separation of the combustible from the non-combustible fraction of 1.5 tons of MSW per day. 


$$
\text { APPENDIX - I. }
$$

\section{BIBLIOGRAPHY}

1. Bartlett, J. (1983) "The Effect of Moisture of Air Classification of Municipal Solid Waste," Thesis Presented to Duke University.

2. Crowe, P.B., and J.J. Peirce (1988) "Particle Density and Air-classifier Performance," J. Energy Engra., 114(2), 382-399.

3. Everett, J. and J.J. Peirce (1989a) "Variable Injection Active-Pulse Air Classification," J. Envir. Engrg., ASCE $115(2), 395-408$.

4. Everett, J. and J.J. Peirce (1989b) "The Effect Feed Rate and Classifier Height on the Air classification of Municiple Solid Waste," J. Envir. Engrg. accepted for publication.

5. Jackson, C.R., R. Stessel, and J:J. Peirce (1988) "Passive Pulsing Air Classifier Theory," J. Envir. Engrg., 114(1), 106-119.

6. Peirce, J.J., (1988) "Pulsed Air Classification for Resource Recovery and Energy Pxoduction," ISWA 88 proceedings, Volume $1,351-357$.

7. Peirce, J.J., R. Stessel, and B. Wolf (1986) "PassivePulsing Air Classifiers for Energy Production," I. Energy Engrg., 112 (1) 25-36.

8. Peirce, J.J. and R. Stessel (1983) "Pulsed Flow Air Classification for Waste-to-Energy," J. Energy Engrg., $109(1), 60-73$.

9. Peirce, J.J., P.A. Vesilind, and M. McNabb (1983) "Predicting Air Classifier Performance," J. Energy Engrg., 109 (1), 7-16.

10. Peirce, J.J., P.A. Vesilind, and R. Stessel (1983) "Quantifying' the Performance of $\mathrm{N}$-Way Separators," Resources and Conservation, 10(3), 243-248.

11. Peirce, J.J. and N. Wittenberg (1984) "Zig-Zag Configurations and Air Classifier Performance," J. Energy Engrg., $110(1), 36-48$. 
12. Saul, C. (1981) "Air Classification of Glass Particles," Thesis Presented to Duke University in Partial Fulfillment of the Requirements for the Degree of Master of Science.

13. Stessel, R. and J.J. Peirce (1987) "Particle Separation in Pulsed Air Flow," J. Eng. Mech., 113(10), 1594-1607.

14. Stessel, R. and J.J. Peirce, (1986) "Comparing Pulsing Classifiers for Waste-to-Energy," J. Energy Engrg., ASCE, $112(1), 1-13$.

5. Stessel, R. and J.J. Peirce (1985) "Separation of Solid Waste with Pulsed Airflow," J. Envir. Engrg., 111(6), 833849 .

16. Stessel, R., J.J. Peirce, and Wolf, B. (1986) "Passive Pulsing Air Classifiers for Energy Production," J. Energy Engrg., ASCE $112(1), 25-36$.

17. Stessel, R. (1983) "Pulsed Flow Air Classification," Thes is Presented to Duke University, Durham, N.C., in Partial Fulfillment of the Requirements for the Degree of Doctor of Philosophy.

18. Stessel, R. and J.J..Peirce (1983) "Pulsed-flow Air Classification for Waste-to-Energy," J. Energy Engrg., $109(2), 60-73$.

19. Taub, B. and J.J. Peirce (1983) "Instabilities in Air Classification of Fuels," J. Energy Engrg., 109(2), 74-87.

20. Vesilind, P.A. (1.986) "Air Classification of Shredder Refuse," Conservation and Recycling, 9(1), 35-44.

21. Vesilind, P.A. and R.A. Henrikson (1981) "Effect of Feed Rate on Air Classifier Performance," Resources and Conservation, 6(3-4), 211-221.

22. Vesilind, P.A., J.J. Peirce, and M. McNabb (1982)

"Predicting Particle Behavior in Air Classifiers," conservation and Recycling, 5(4), 209-213. 


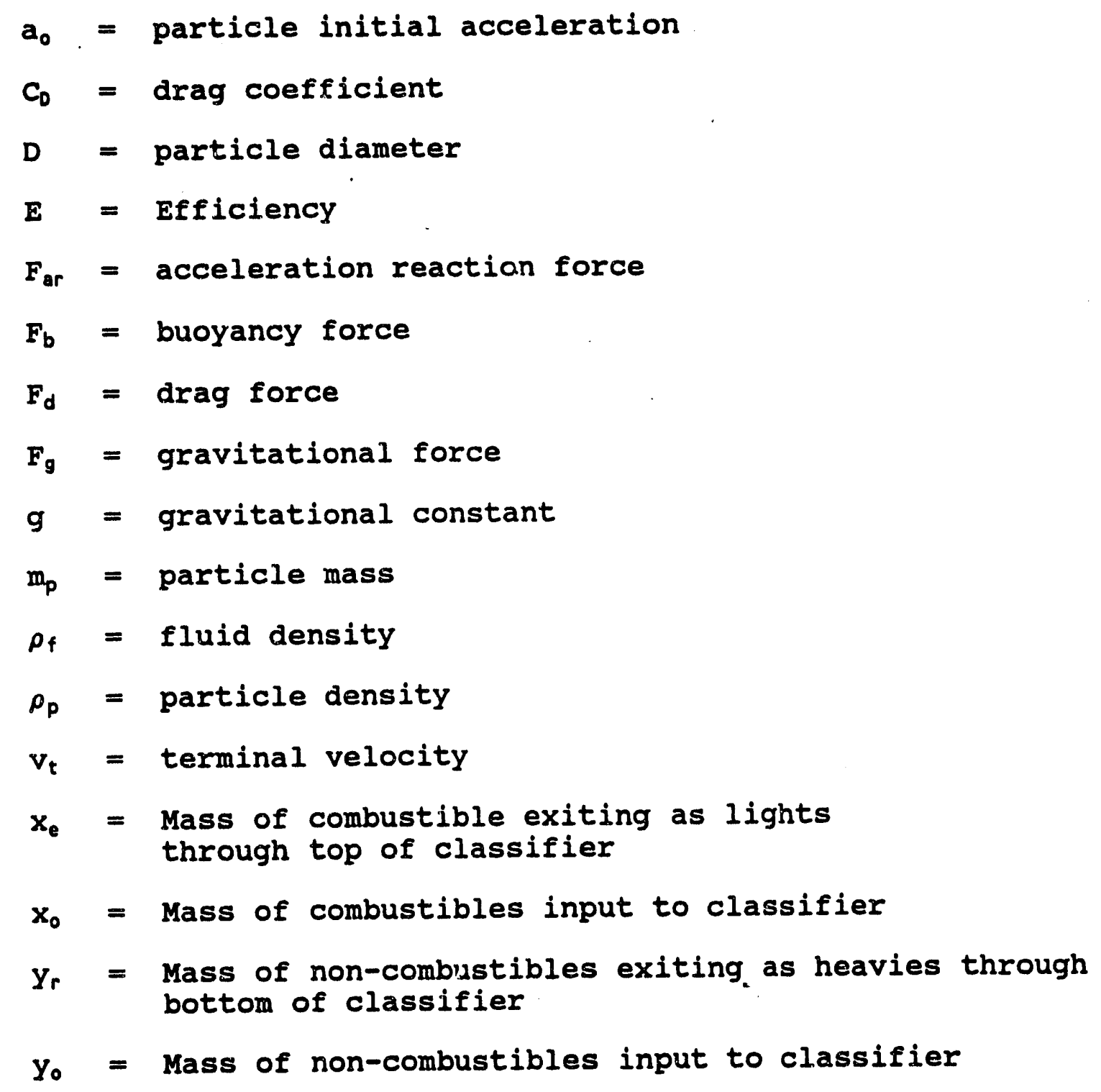




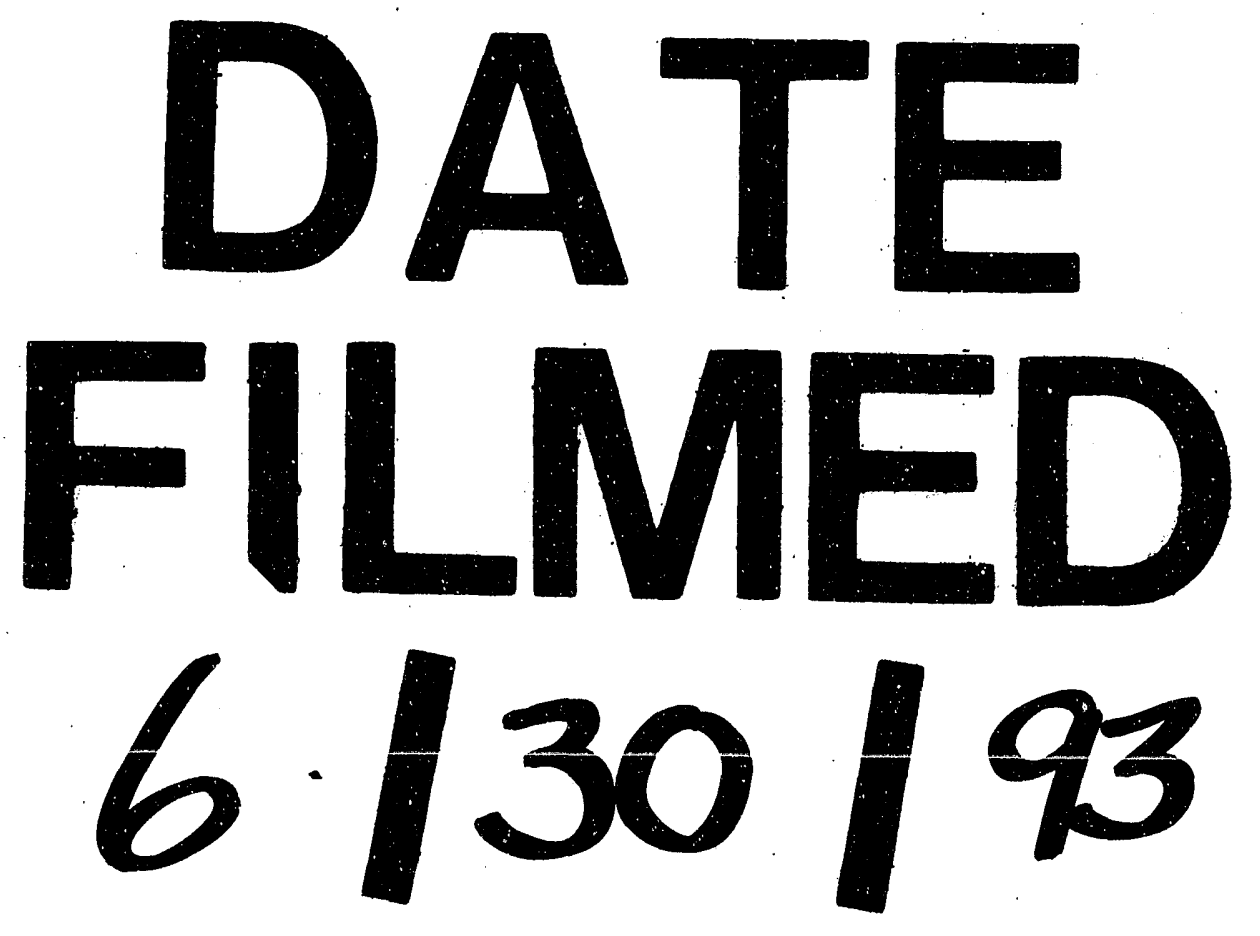


